\title{
CURVATURE PINCHING THEOREMS FOR MINIMAL SURFACES IN COMPLEX GRASSMANN MANIFOLDS
}

\author{
By \\ Wu BING-YE
}

\begin{abstract}
In this paper we study the curvature pinching property for minimal surfaces in complex Grassmann manifolds and obtain some results.
\end{abstract}

\section{Introduction}

Harmonic maps and minimal immersions of a Riemann surface $M$ into complex projective space $C P^{n}$ and complex Grassmann manifold $G(m, n)$ have been studied from a variety of viewpoints (see e.g. $[1-5,7]$ ), and the basic work of which was established by Chern and Wolfson in [3, 4]. For minimal surfaces in $C P^{n}$ there is an invariant $\alpha$ called the Kaehler angle which is related to the complex structure $J$ of $C P^{n}$. The Kaehler angle, whose importance in the theory of minimal surfaces in Kaehler manifolds was pointed by Chern and Wolfson [3], gives a measure of the failure of the immersion to be a holomorphic map. For harmonic isometric immersions from surfaces into complex Grassmann manifolds, or equivalently, for minimal surfaces in complex Grassmann manifolds, there is an analogous invariant i.e., the Kaehler angle, and we shall use this invariant to study the curvature pinching property for harmonic isometric immersions from surfaces into complex Grassmann manifolds.

\section{Preliminaries}

A. The Geometry of $G(m, n)$

We equip $C^{n}$ with the standard Hermitian inner product, so that, for $Z$, $W \in C^{n}$,

$$
Z=\left(z_{1}, \ldots, z_{n}\right), \quad W=\left(w_{1}, \ldots, w_{n}\right)
$$

Received September 20, 1999

Revised March 13, 2000 
we have

$$
\langle Z, W\rangle=\sum_{A} z_{A} \bar{w}_{A}
$$

here and later on we agree on the following convension of the ranges of indices:

$$
1 \leq A, B, \ldots \leq n, \quad 1 \leq \alpha, \beta, \ldots \leq m, \quad m+1 \leq i, j, \ldots \leq n
$$

A frame consists of an ordered set of $n$ linearly independent vectors $Z_{A}$ so that

$$
Z_{1} \wedge \cdots \wedge Z_{n} \neq 0 \text {. }
$$

It is called unitary, if

$$
\left\langle Z_{A}, Z_{B}\right\rangle=\delta_{A B}
$$

The space of unitary frames can be identified with the unitary group $U(n)$. With

$$
d Z_{A}=\sum_{B} \omega_{A B} Z_{B}
$$

the forms $\omega_{A B}$ are the Maurer-Cartan forms of $U(n)$. They are skew-Hermitian, i.e., we have

$$
\omega_{A B}+\bar{\omega}_{B A}=0
$$

Taking the exterior derivative of (2.1), we get the Maurer-Cartan equations of $U(n)$ :

$$
d \omega_{A B}=\sum_{C} \omega_{A C} \wedge \omega_{C B}
$$

Let $G(m, n)$ be the complex Grassmann manifold of all $m$-dimensional subspace $C^{m}$ in $C^{n}$. An element $C^{m}$ of $G(m, n)$ can be defined by the multivector $Z_{1} \wedge \cdots \wedge Z_{m} \neq 0$, defined up to a factor. The vectors $Z_{\alpha}$ and their orthogonal vectors $Z_{i}$ are defined up to a transformation of $U(m)$ and $U(n-m)$, respectively, so that $G(m, n)$ has a $G$-structure, with $G=U(m) \times U(n-m)$. In particular, the form

$$
d s^{2}=\sum_{\alpha, i} \omega_{\alpha i} \bar{\omega}_{\alpha i}
$$

is a positive definite Hermitian form on $G(m, n)$, which defines a canonical Hermitian metric. It is easy to see that $d s^{2}$ is infact a Kaehler metric on $G(m, n)$. Note that when $m=1$, this induced metric on $G(1, n)=C P^{n-1}$ has constant 
holomorphic sectional curvature 4 . Let

$$
\omega_{\alpha i}=\theta_{\alpha i}+\sqrt{-1} \theta_{\alpha^{*} i^{*}}
$$

i.e., $\theta_{\alpha i}$ and $\theta_{\alpha^{*} i^{*}}$ are the real and imaginary parts of $\omega_{\alpha i}$ respectively. Let $\left\{E_{\alpha i}, E_{\alpha^{*} i^{*}}\right\}$ be the dual frame of $\left\{\theta_{\alpha i}, \theta_{\alpha^{*} i^{*}}\right\}$. The complex structure $J$ of $G(m, n)$ is defined by

$$
J E_{\alpha i}=E_{\alpha^{*} i^{*}}, \quad J E_{\alpha^{*} i^{*}}=-E_{\alpha i}
$$

so that $\left\{E_{\alpha i}, E_{\alpha^{*} i^{*}}\right\}$ is the $J$-canonical frame of $G(m, n)$.

\section{B. Harmonic Maps from Surfaces into $G(m, n)$}

Let $M$ be an oriented Riemannian surface and $f: M \rightarrow G(m, n)$ be a nonconstant harmonic map. Let $e_{1}, e_{2}$ be a local orthonormal frame of $M$ adapted to the orientation, and $\theta_{1}, \theta_{2}$ be the dual frame. The structure equations of $M$ are

$$
d e_{1}=\rho e_{2}, \quad d e_{2}=-\rho e_{1}, \quad d \rho=-\kappa \theta_{1} \wedge \theta_{2},
$$

where $\rho$ is the real connection form and $\kappa$ is the Gaussian curvature of $M$. If we set $\varphi=\theta_{1}+\sqrt{-1} \theta_{2}$, then we have the following complex version of (2.7):

$$
d \varphi=-\sqrt{-1} \rho \wedge \varphi, \quad d \rho=-\frac{\sqrt{-1}}{2} \kappa \varphi \wedge \bar{\varphi}
$$

Let

$$
f^{*}\left(\omega_{\alpha i}\right)=a_{\alpha i} \varphi+b_{\alpha i} \bar{\varphi} .
$$

Since $d s_{M}^{2}=\varphi \bar{\varphi}$, it is easy to know that $f$ is an isometric immersion if and only if

$$
\sum_{\alpha, i} a_{\alpha i} \bar{b}_{\alpha i}=0, \quad \sum_{\alpha, i}\left(a_{\alpha i} \bar{a}_{\alpha i}+b_{\alpha i} \bar{b}_{\alpha i}\right)=1
$$

In terms of $a_{\alpha i}$ and $b_{\alpha i}$ Chern and Wolfson defined the so-called $\partial$ - and $\bar{\partial}$-transformations as follows [4].

$$
\begin{gathered}
\partial f: M \rightarrow G\left(m_{1}, n\right), \quad \partial f(x)=\operatorname{span}\left\{\sum_{i} a_{\alpha i} Z_{i}: 1 \leq \alpha \leq m\right\}, \quad \text { if } \sum_{\alpha, i}\left|a_{\alpha i}\right|^{2} \neq 0, \\
\bar{\partial} f: M \rightarrow G\left(m_{-1}, n\right), \quad \bar{\partial} f(x)=\operatorname{span}\left\{\sum_{i} b_{\alpha i} Z_{i}: 1 \leq \alpha \leq m\right\}, \quad \text { if } \sum_{\alpha, i}\left|b_{\alpha i}\right|^{2} \neq 0,
\end{gathered}
$$

where $m_{1}$ and $m_{-1}$ are positive integers, called the ranks of $\partial f$ and $\bar{\partial} f$ respectively. For convenience we will drop $f^{*}$ in such formulae when its presence is 
clear from context. Taking the exterior derivative of (2.9) and using (2.3) and $(2.8)$, we see that there exist locally defined complex-valued functions $p_{x i}, q_{x i}, r_{x i}$ such that [4]

$$
\begin{aligned}
& p_{x i} \varphi+q_{x i} \bar{\varphi}=d a_{x i}-\sum_{\beta} a_{\beta i} \omega_{\alpha \beta}+\sum_{j} a_{x j} \omega_{j i}-\sqrt{-1} a_{x i} \rho \\
& q_{x i} \varphi+r_{x i} \bar{\varphi}=d b_{x i}-\sum_{\beta} b_{\beta i} \omega_{\alpha \beta}+\sum_{j} b_{\alpha j} \omega_{j i}+\sqrt{-1} b_{x i} \rho .
\end{aligned}
$$

The quadratic differential form $p_{x i} \varphi^{2}+2 q_{x i} \varphi \bar{\varphi}+r_{x i} \bar{\varphi}^{2}$ is the complex version of the second fundamental form of $f$. It is well-known that the vanishing of its trace is the condition that $f$ be harmonic, so that $q_{x i}=0$, c.f. [4]. By [7] we know that the quantities $\sum_{x, i}\left|a_{x i}\right|^{2}$ and $\sum_{x, i}\left|b_{x i}\right|^{2}$ are globally defined invariants on $M$, and we shall give the geometric meanings of them. Let

$$
\begin{gathered}
f^{*}\left(\theta_{x i}\right)=c_{x i 1} \theta_{1}+c_{x i 2} \theta_{2}, \\
f^{*}\left(\theta_{\alpha^{*} i^{*}}\right)=c_{\alpha^{*} i^{*} 1} \theta_{1}+c_{\alpha^{*} i^{*} 2} \theta_{2} .
\end{gathered}
$$

Then from (2.5) and (2.9) we get

$$
\begin{gathered}
c_{\alpha i 1}=\frac{1}{2}\left(a_{\alpha i}+\bar{a}_{x i}+b_{\alpha i}+\bar{b}_{\alpha i}\right), \\
c_{\alpha i 2}=\frac{\sqrt{-1}}{2}\left(a_{x i}-\bar{a}_{x i}-b_{\alpha i}+\bar{b}_{\alpha i}\right), \\
c_{x^{*} i^{*} 1}=\frac{\sqrt{-1}}{2}\left(-a_{x i}+\bar{a}_{x i}-b_{\alpha i}+\bar{b}_{\alpha i}\right), \\
c_{\alpha^{*} i^{*} 2}=\frac{1}{2}\left(a_{x i}+\bar{a}_{x i}-b_{\alpha i}-\bar{b}_{\alpha i}\right) .
\end{gathered}
$$

From (2.12) we can easily get

$$
\begin{aligned}
& f_{*}\left(e_{1}\right)=\sum_{\alpha, i}\left(c_{\alpha i 1} E_{\alpha i}+c_{a^{*} i^{*} 1} E_{\alpha^{*} i^{*}}\right), \\
& f_{*}\left(e_{2}\right)=\sum_{\alpha, i}\left(c_{\alpha i 2} E_{\alpha i}+c_{\alpha^{*} i^{*} 2} E_{\alpha^{*} i^{*}}\right) .
\end{aligned}
$$

If $f$ is conformal, i.e., $\sum_{\alpha, i} a_{x i} \bar{b}_{x i}=0$, then from (2.6), (2.13) and (2.14) we see that the cosin of the angle between $J f_{*}\left(e_{1}\right)$ and $f_{*}\left(e_{2}\right)$ is

$$
\cos \alpha=\frac{\left\langle J f_{*}\left(e_{1}\right), f_{*}\left(e_{2}\right)\right\rangle}{\mid\left\langle J f_{*}\left(e_{1}\right)|\cdot| f_{*}\left(e_{2}\right)\right|}=\frac{\sum_{x, i}\left(\left|a_{x i}\right|^{2}-\left|b_{\alpha i}\right|^{2}\right)}{\sum_{\alpha, i}\left(\left|a_{x i}\right|^{2}+\left|b_{x i}\right|^{2}\right)},
$$


which is an invariant on $M$. Therefore, for conformal harmonic map $f: M \rightarrow G(m, n)$, we can define the Kaehler function $\cos \alpha$ and Kaehler angle $\alpha$ as above. In particular, when $M=S^{2}$, the topological 2-sphere, all harmonic maps from $S^{2}$ into $G(m, n)$ are always conformal [4, 7]. Thus for harmonic 2-sphere in $G(m, n)$, there is an invariant $\alpha$, called the Kaehler angle, which gives a measure of the failure of $f$ to be a holomorphic map. Now we assume that $f: M \rightarrow G(m, n)$ is a harmonic isometric immersion, i.e., an isometric minimal immersion from $M$ into $G(m, n)$. Then (2.10) and (2.15) yields

$$
\sum_{\alpha, i}\left|a_{\alpha i}\right|^{2}=\cos ^{2} \frac{\alpha}{2}, \quad \sum_{\alpha, i}\left|b_{\alpha i}\right|^{2}=\sin ^{2} \frac{\alpha}{2},
$$

which gives the geometric meanings of $\sum_{\alpha, i}\left|a_{\alpha i}\right|^{2}$ and $\sum_{\alpha, i}\left|b_{\alpha i}\right|^{2}$. As in the special case of $m=1$, we call the isometric minimal immersion $f: M \rightarrow G(m, n)$ is holomorphic, anti-holomorphic and totally real if $\alpha=0, \pi$ and $\pi / 2$ respectively. It is clear that $f$ is holomorphic if and only if $\bar{\partial} f=0$, while $f$ is anti-holomorphic if and only if $\partial f=0$. In terms of matrix notation we collect the fundamental formulae for a minimal isometric immersion $f: M \rightarrow G(m, n)$ as follows.

$$
\begin{gathered}
\operatorname{tr}\left(A \bar{B}^{t}\right)=0, \quad\|A\|^{2}=\cos ^{2} \frac{\alpha}{2}, \quad\|B\|^{2}=\sin ^{2} \frac{\alpha}{2} \\
d A-\phi_{11} A+A \phi_{22}-\sqrt{-1} A \rho=P \varphi \\
d B-\phi_{11} B+B \phi_{22}+\sqrt{-1} B \rho=R \bar{\varphi}
\end{gathered}
$$

where

$$
\begin{gathered}
A=\left(\begin{array}{ccc}
a_{1, m+1} & \cdots & a_{1 n} \\
\vdots & & \vdots \\
a_{m, m+1} & \cdots & a_{m n}
\end{array}\right), \quad B=\left(\begin{array}{ccc}
b_{1, m+1} & \cdots & b_{1 n} \\
\vdots & & \vdots \\
b_{m, m+1} & \cdots & b_{m n}
\end{array}\right), \\
P=\left(\begin{array}{ccc}
p_{1, m+1} & \cdots & p_{1 n} \\
\vdots & & \vdots \\
p_{m, m+1} & \cdots & p_{m n}
\end{array}\right), \quad R=\left(\begin{array}{ccc}
r_{1, m+1} & \cdots & r_{1 n} \\
\vdots & & \vdots \\
r_{m, m+1} & \cdots & r_{m n}
\end{array}\right), \\
\phi_{11}=\left(\begin{array}{ccc}
\omega_{11} & \cdots & \omega_{1 m} \\
\vdots & & \vdots \\
\omega_{m 1} & \cdots & \omega_{m m}
\end{array}\right), \quad \phi_{22}=\left(\begin{array}{ccc}
\omega_{m+1, m+1} & \cdots & \omega_{m+1, n} \\
\vdots & & \vdots \\
\omega_{n, m+1} & \cdots & \omega_{n n}
\end{array}\right) .
\end{gathered}
$$

Here we define the norm $\|C\|$ of a matrix $C$ by $\|C\|^{2}=\operatorname{tr}\left(C \bar{C}^{t}\right)$ in a standard way, $\alpha$ is the Kaehler angle of $f$. 


\section{Curvature Pinching Theorems for Minimal Surfaces}

In this section we shall study the curvature pinching property for a minimal isometric immersion $f: M \rightarrow G(m, n)$. For this porpose let us first prove the following

LEMMA 3.1. Let $f: M \rightarrow G(m, n)$ be an isometric minimal immersion from an oriented Riemannian surface $M$ into $G(m, n)$. Then we have the following Gauss equation for $f$ :

$$
\kappa=4\left(\left\|A \bar{A}^{t}\right\|^{2}+\left\|B \bar{B}^{t}\right\|^{2}-\left\|\bar{A}^{t} B\right\|^{2}-\left\|A \bar{B}^{t}\right\|^{2}\right)-2\left(\|P\|^{2}+\|R\|^{2}\right) .
$$

Proof. By (2.3) and (2.9) we have

$$
\begin{aligned}
& d \phi_{11}=\phi_{11} \wedge \phi_{11}+\left(B \bar{B}^{t}-A \bar{A}^{t}\right) \varphi \wedge \bar{\varphi} \\
& d \phi_{22}=\phi_{22} \wedge \phi_{22}+\left(\bar{A}^{t} A-\bar{B}^{t} B\right) \varphi \wedge \bar{\varphi}
\end{aligned}
$$

Taking the exterior derivative of $(2.17)_{2-3}$ and using (2.8) and (3.2) we get

$$
\begin{aligned}
& d P-\phi_{11} P+P \phi_{22}-2 \sqrt{-1} P \rho=P_{, 1} \varphi+P_{, 2} \bar{\varphi}, \\
& \text { with } P_{, 2}=\frac{1}{2} \kappa A+B \bar{B}^{t} A+A \bar{B}^{t} B-2 A \bar{A}^{t} A ; \\
& d R-\phi_{11} R+R \phi_{22}+2 \sqrt{-1} R \rho=R_{, 1} \varphi+R_{, 2} \bar{\varphi}, \\
& \text { with } \quad R_{, 1}=\frac{1}{2} \kappa B+A \bar{A}^{t} B+B \bar{A}^{t} A-2 B \bar{B}^{t} B .
\end{aligned}
$$

From $(2.17)_{2}$ we can calculate out that

$$
d\left(A \bar{A}^{t}\right)-\phi_{11} A \bar{A}^{t}+A \bar{A}^{t} \phi_{11}=P \bar{A}^{t} \varphi+A \bar{P}^{t} \bar{\varphi} .
$$

So, by taking the trace on both sides of (3.4) we get

$$
d \cos ^{2} \frac{\alpha}{2}=d \operatorname{tr}\left(A \bar{A}^{t}\right)=\operatorname{tr}\left(P \bar{A}^{t}\right) \varphi+\operatorname{tr}\left(A \bar{P}^{t}\right) \bar{\varphi} .
$$

By virtue of (2.17), (3.3) and (3.5) we obtain

$$
\begin{aligned}
\frac{1}{4} \Delta \cos ^{2} \frac{\alpha}{2} \varphi \wedge \bar{\varphi} & =\partial \bar{\partial} \cos ^{2} \frac{\alpha}{2}=d\left(\bar{\partial} \cos ^{2} \frac{\alpha}{2}\right)=d\left(\operatorname{tr}\left(A \bar{P}^{t}\right) \bar{\varphi}\right) \\
& =\left(\frac{1}{2} \cos ^{2} \frac{\alpha}{2} \cdot \kappa-2\left\|A \bar{A}^{t}\right\|^{2}+\|P\|^{2}+\left\|\bar{A}^{t} B\right\|^{2}+\left\|A \bar{B}^{t}\right\|^{2}\right) \varphi \wedge \bar{\varphi}
\end{aligned}
$$


where $\Delta$ denotes the Laplacian of $M$. Similarly we have

$$
\frac{1}{4} \Delta \sin ^{2} \frac{\alpha}{2}=\frac{1}{2} \sin ^{2} \frac{\alpha}{2} \cdot \kappa-2\left\|B \bar{B}^{t}\right\|^{2}+\|R\|^{2}+\left\|\bar{A}^{t} B\right\|^{2}+\left\|A \bar{B}^{t}\right\|^{2} .
$$

Combining (3.6) and (3.7) we can get (3.1) immediately.

Using this Gauss equation, we can prove the following

THEOREM 3.2. Let $f: M \rightarrow G(m, n)$ be an isometric minimal immersion of a connected surface (not necessary complete) $M$ into $G(m, n)$. If $\kappa \geq$ $\max \left\{4 \cos ^{2}(\alpha / 2), 4 \sin ^{2}(\alpha / 2)\right\}$, then one of the following two cases holds:

(a) $\kappa=4$, and $f$ is either holomorphic or anti-holomorphic;

(b) $\kappa=2, f$ is totally real, and $f=\left[f_{1}+V\right]$, where $f_{1}: M \rightarrow G(2, n)$ is a totally real isometric minimal immersion, and $V$ is a constant complex vector subspace of $C^{n}$ with dimension $m-2$.

Proof. It is clear that

$$
\left\|A \bar{A}^{t}\right\|^{2} \leq\|A\|^{4}=\cos ^{4} \frac{\alpha}{2}
$$

and the equality holds if and only if $\operatorname{rank}(A)=\operatorname{rank}(\partial f) \leq 1$. Similarly,

$$
\left\|B \bar{B}^{t}\right\|^{2} \leq\|B\|^{4}=\sin ^{4} \frac{\alpha}{2},
$$

and the equality holds if and only if $\operatorname{rank}(B)=\operatorname{rank}(\bar{\partial} f) \leq 1$. Therefore, from (3.1), (3.8) and (3.9) we have

$$
\begin{aligned}
0= & \kappa-4\left(\left\|A \bar{A}^{t}\right\|^{2}+\left\|B \bar{B}^{t}\right\|^{2}\right)+4\left(\left\|\bar{A}^{t} B\right\|^{2}+\left\|A \bar{B}^{t}\right\|^{2}\right)+2\left(\|P\|^{2}+\|R\|^{2}\right) \\
\geq & \cos ^{2} \frac{\alpha}{2}\left(\kappa-4 \cos ^{2} \frac{\alpha}{2}\right)+\sin ^{2} \frac{\alpha}{2}\left(\kappa-4 \sin ^{2} \frac{\alpha}{2}\right) \\
& +4\left(\left\|\bar{A}^{t} B\right\|^{2}+\left\|A \bar{B}^{t}\right\|^{2}\right)+2\left(\|P\|^{2}+\|R\|^{2}\right)
\end{aligned}
$$

and the equality holds if and only if $\operatorname{rank}(\partial f) \leq 1, \operatorname{rank}(\bar{\partial} f) \leq 1$. By $(3.10)$ and the curvature condition it is clear that if $f$ is not totally real, then $f$ must be holomorphic or anti-holomorphic with $\kappa=4$, which is contained in case (a) of the theorem. Now we consider the case when $f$ is totally real. Then by (3.10) we have $\kappa=2$, and

$$
\bar{A}^{t} B=0, \quad A \bar{B}^{t}=0, \quad P=R=0, \quad \operatorname{rank}(\partial f)=\operatorname{rank}(\bar{\partial} f)=1 .
$$

So we can choose the local unitary frame $Z_{1}, \ldots, Z_{n}$ of $C^{n}$ along $f$ suitably so 
that $f=\left[Z_{1} \wedge \cdots \wedge Z_{m}\right], \quad \partial f=\left[Z_{m+1}\right]$ and $\operatorname{ker}(\partial f)=\left[Z_{2} \wedge \cdots \wedge Z_{m}\right]$. Furthermore, since $A \bar{B}^{t}=0$ implies that $\partial f \perp \bar{\partial} f$, we can require that $\bar{\partial} f=\left[Z_{m+2}\right]$ and $\operatorname{ker}(\partial f) \cap \operatorname{ker}(\bar{\partial} f)=\left[Z_{3} \wedge \cdots \wedge Z_{m}\right]$. In summary, the pull back of the Maurer-Cartan form of $U(n)$ by $f$ is given by

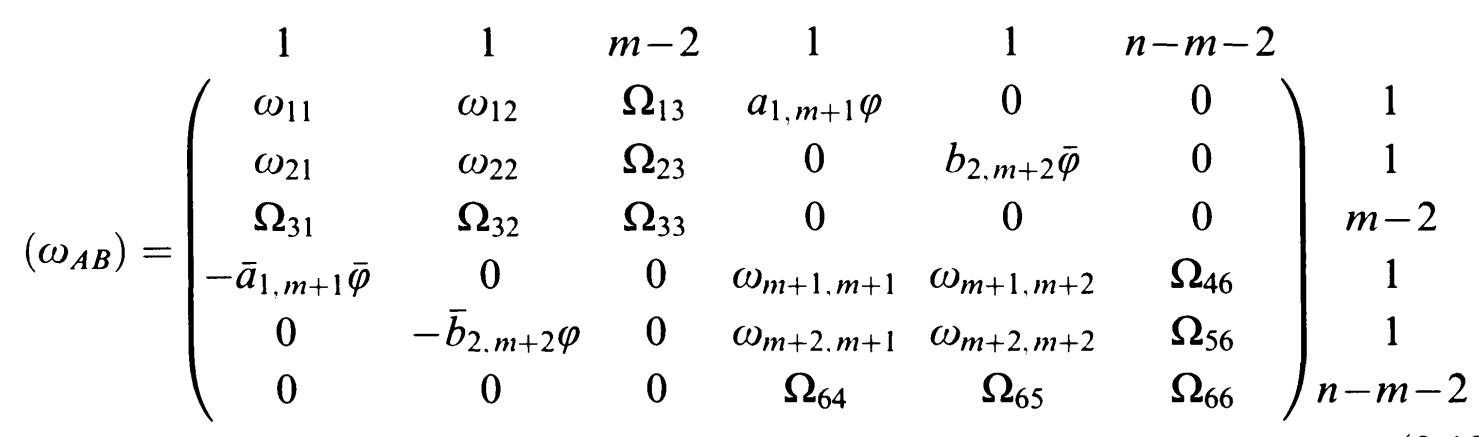

By virtue of $(2.17)_{2-3},(3.11)$ and (3.12) we get

$$
\begin{aligned}
& d\left(\begin{array}{ccc}
a_{1, m+1} & 0 & 0 \\
0 & 0 & 0 \\
0 & 0 & 0
\end{array}\right)-\left(\begin{array}{lll}
\omega_{11} & \omega_{12} & \Omega_{13} \\
\omega_{21} & \omega_{22} & \Omega_{23} \\
\Omega_{31} & \Omega_{32} & \Omega_{33}
\end{array}\right)\left(\begin{array}{ccc}
a_{1, m+1} & 0 & 0 \\
0 & 0 & 0 \\
0 & 0 & 0
\end{array}\right) \\
& +\left(\begin{array}{ccc}
a_{1, m+1} & 0 & 0 \\
0 & 0 & 0 \\
0 & 0 & 0
\end{array}\right)\left(\begin{array}{ccc}
\omega_{m+1, m+1} & \omega_{m+1, m+2} & \Omega_{46} \\
\omega_{m+2 . m+1} & \omega_{m+2, m+2} & \Omega_{56} \\
\Omega_{64} & \Omega_{65} & \Omega_{66}
\end{array}\right) \\
& -\sqrt{-1} \rho\left(\begin{array}{ccc}
a_{1, m+1} & 0 & 0 \\
0 & 0 & 0 \\
0 & 0 & 0
\end{array}\right)=0 \\
& d\left(\begin{array}{ccc}
0 & 0 & 0 \\
0 & b_{2, m+2} & 0 \\
0 & 0 & 0
\end{array}\right)-\left(\begin{array}{ccc}
\omega_{11} & \omega_{12} & \Omega_{13} \\
\omega_{21} & \omega_{22} & \Omega_{23} \\
\Omega_{31} & \Omega_{32} & \Omega_{33}
\end{array}\right)\left(\begin{array}{ccc}
0 & 0 & 0 \\
0 & b_{2, m+2} & 0 \\
0 & 0 & 0
\end{array}\right) \\
& +\left(\begin{array}{ccc}
0 & 0 & 0 \\
0 & b_{2, m+2} & 0 \\
0 & 0 & 0
\end{array}\right)\left(\begin{array}{ccc}
\omega_{m+1, m+1} & \omega_{m+1, m+2} & \Omega_{46} \\
\omega_{m+2, m+1} & \omega_{m+2, m+2} & \Omega_{56} \\
\Omega_{64} & \Omega_{65} & \Omega_{66}
\end{array}\right) \\
& +\sqrt{-1} \rho\left(\begin{array}{ccc}
0 & 0 & 0 \\
0 & b_{2, m+2} & 0 \\
0 & 0 & 0
\end{array}\right)=0
\end{aligned}
$$


from which it follows that

$$
\Omega_{31}=\Omega_{32}=0 .
$$

Put $f_{1}=\left[Z_{1} \wedge Z_{2}\right]: M \rightarrow G(2, n)$ and $V=\left[Z_{3} \wedge \cdots \wedge Z_{m}\right]$. Then by (3.12) and (3.14), we can get the conclusion of the theorem easily.

The following theorem is a generalization of Theorem 2.1 of [7].

THEOREM 3.3. Let $f: M \rightarrow G(m, n)$ be an isometric minimal immersion of a compact surface $M$ into $G(m, n)$ which is not anti-holomorphic (resp. not holomorphic). If $\kappa \geq 4 \cos ^{2}(\alpha / 2) / \operatorname{rank}(\partial f) \quad\left(\right.$ resp. $\left.\quad \kappa \geq 4 \sin ^{2}(\alpha / 2) / \operatorname{rank}(\bar{\partial} f)\right)$, then $\kappa=4 \cos ^{2}(\alpha / 2) / \operatorname{rank}(\partial f)\left(\operatorname{resp} . \quad \kappa=4 \sin ^{2}(\alpha / 2) / \operatorname{rank}(\bar{\partial} f)\right)$.

Proof. We will give the proof for the case $\kappa \geq 4 \cos ^{2}(\alpha / 2) / \operatorname{rank}(\partial f)$ only because the other can be shown similarly. As in the proof of Theorem 3.2, we can choose a local unitary frame $Z_{1}, \ldots, Z_{n}$ of $C^{n}$ along $f$ suitably so that the pull back of the Maurer-Cartan form of $U(n)$ by $f$ is given by

$$
\left(\omega_{A B}\right)=\left(\begin{array}{cccc}
k_{1} & m-k_{1} & k_{1} & n-m-k_{1} \\
\Omega_{11} & \Omega_{12} & A_{11} \varphi+B_{11} \bar{\varphi} & B_{12} \bar{\varphi} \\
\Omega_{21} & \Omega_{22} & B_{21} \bar{\varphi} & B_{22} \bar{\varphi} \\
-\bar{A}_{11}^{t} \bar{\varphi}-\bar{B}_{11}^{t} \varphi & -\bar{B}_{21}^{t} \varphi & \Omega_{33} & \Omega_{34} \\
-\bar{B}_{12}^{t} \varphi & -\bar{B}_{22}^{t} \varphi & \Omega_{43} & \Omega_{44}
\end{array}\right) k_{1} k_{1},
$$

where $k_{1}=\operatorname{rank}(\partial f) .(2.17)_{2}$ and (3.15) yields

$$
\begin{gathered}
d\left(\begin{array}{cc}
A_{11} & 0 \\
0 & 0
\end{array}\right)-\left(\begin{array}{ll}
\Omega_{11} & \Omega_{12} \\
\Omega_{21} & \Omega_{22}
\end{array}\right)\left(\begin{array}{cc}
A_{11} & 0 \\
0 & 0
\end{array}\right)+\left(\begin{array}{cc}
A_{11} & 0 \\
0 & 0
\end{array}\right)\left(\begin{array}{ll}
\Omega_{33} & \Omega_{34} \\
\Omega_{43} & \Omega_{44}
\end{array}\right) \\
-\sqrt{-1} \rho\left(\begin{array}{cc}
A_{11} & 0 \\
0 & 0
\end{array}\right)=\left(\begin{array}{cc}
P_{11} & P_{12} \\
P_{21} & P_{22}
\end{array}\right) \varphi,
\end{gathered}
$$

from which it follows that

$$
\begin{gathered}
d A_{11}-\Omega_{11} A_{11}+A_{11} \Omega_{33}-\sqrt{-1} \rho A_{11}=P_{11} \varphi \\
A_{11} \Omega_{34}=P_{12} \varphi, \quad \Omega_{21} A_{11}=P_{21} \varphi, \quad P_{22}=0 .
\end{gathered}
$$

It is easy to see that $\left|\operatorname{det} A_{11}\right|$ is a scalar invariant of $f$ [7]. Noticing that for a nonsingular matrix-valued function $C$ we have $d \log (\operatorname{det} C)=\operatorname{tr}\left(d C \cdot C^{-1}\right)$, we get, from (3.17), 


$$
\begin{gathered}
d\left(\log \operatorname{det} A_{11}\right)-\operatorname{tr}\left(\Omega_{11}\right)+\operatorname{tr}\left(\Omega_{33}\right)-\sqrt{-1} k_{1} \rho=\operatorname{tr}\left(P_{11} A_{11}^{-1}\right) \varphi, \\
\bar{\partial} \log \left|\operatorname{det} A_{11}\right|^{2}=\operatorname{tr}\left(\overline{P_{11} A_{11}^{-1}}\right) \bar{\varphi} .
\end{gathered}
$$

Taking the exterior derivative of $(3.18)_{2}$ and using (2.3), (2.8), (3.15) and (3.17) we obtain

$$
\begin{aligned}
& \Delta \log \left|\operatorname{det} A_{11}\right|^{2} \\
& \quad=2 k_{1} \cdot \kappa+4\left(-2 \cos ^{2} \frac{\alpha}{2}+2\left\|B_{11}\right\|^{2}+\left\|B_{12}\right\|^{2}+\left\|B_{21}\right\|^{2}+\left\|A_{11}^{-1} \boldsymbol{P}_{12}\right\|^{2}+\left\|\boldsymbol{P}_{21} \boldsymbol{A}_{11}^{-1}\right\|^{2}\right) .
\end{aligned}
$$

It should be pointed out that (3.19) holds only in points of $\operatorname{det} A_{11} \neq 0$. However, when $\kappa \geq 4 \cos ^{2}(\alpha / 2) / k_{1}$, by (3.19) and the continuity of $\left|\operatorname{det} A_{11}\right|^{2}$ we can deduce that $\left|\operatorname{det} A_{11}\right|^{2}$ is a subharmonic function on $M$, it must be a constant. Therefore, $\kappa=4 \cos ^{2}(\alpha / 2) / k_{1}$, so we are done.

COROllary $3.4 .^{[7]}$ Let $f: M \rightarrow G(m, n)$ be a harmonic isometric immersion of a compact surface $M$ into $G(m, n)$ which is not anti-holomorphic (resp. not holomorphic). If $\kappa \geq 4 / \operatorname{rank}(\partial f) \quad($ resp. $\kappa \geq 4 / \operatorname{rank}(\bar{\partial} f))$, then $\kappa=4 / \operatorname{rank}(\partial f)$ (resp. $\kappa=4 / \operatorname{rank}(\bar{\partial} f)$ ), and $f$ is holomorphic (resp. anti-holomorphic).

EXAmPle. Let $f: S^{2} \rightarrow G(m, n)$ be defined by

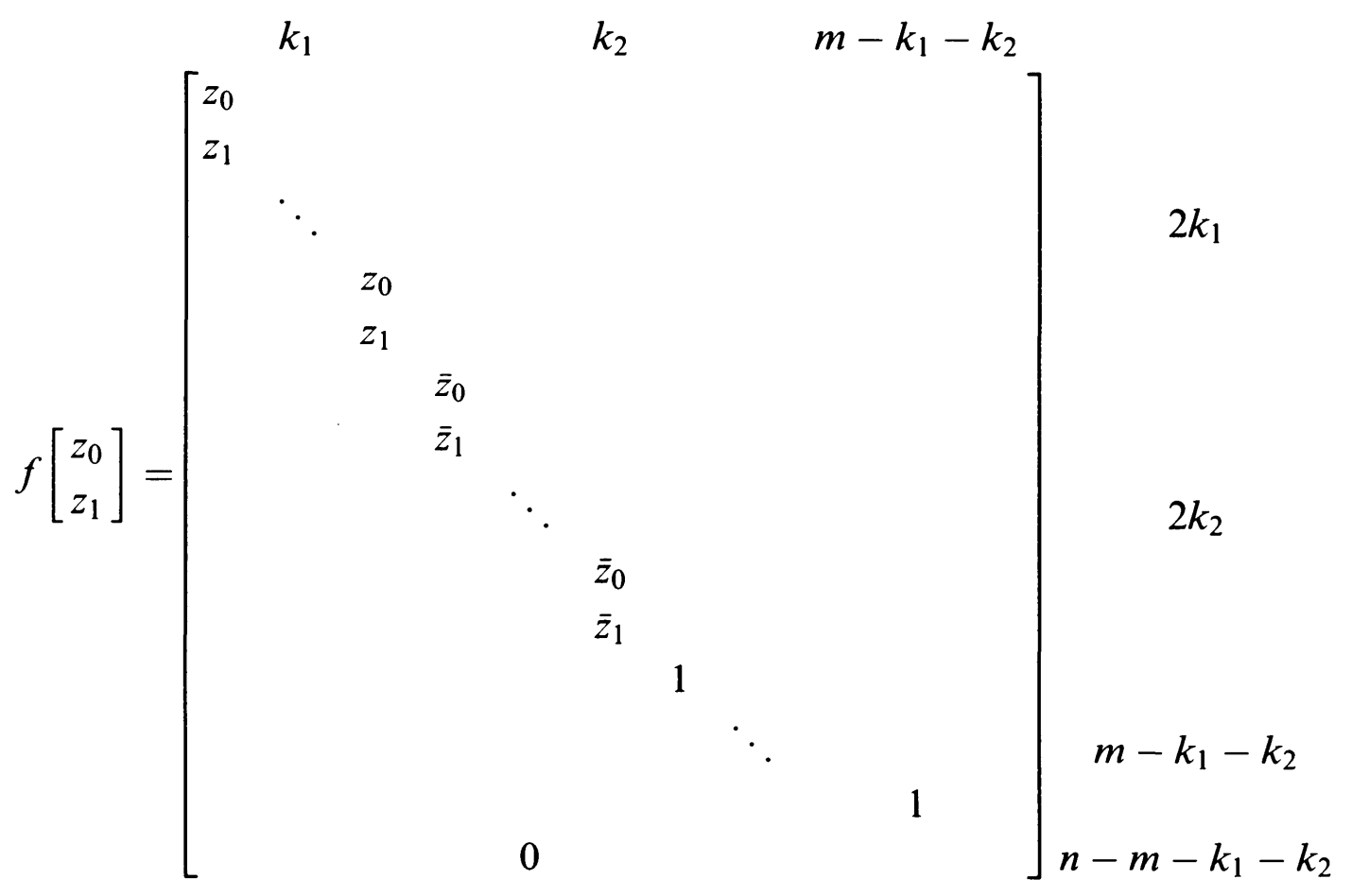


then in the induced metric from that of $G(m, n), S^{2}$ has constant curvature $\kappa=4 /\left(k_{1}+k_{2}\right)$. Also, we have $k_{1}=\operatorname{rank}(\partial f), k_{2}=\operatorname{rank}(\bar{\partial} f), \cos ^{2}(\alpha / 2)=$ $k_{1} /\left(k_{1}+k_{2}\right), \quad$ and $\quad \sin ^{2}(\alpha / 2)=k_{2} /\left(k_{1}+k_{2}\right), \quad$ so $\quad \kappa=4 \cos ^{2}(\alpha / 2) / \operatorname{rank}(\partial f)=$ $4 \sin ^{2}(\alpha / 2) / \operatorname{rank}(\bar{\partial} f)$. Moreover, if $k_{1}=k_{2}=1$, then $\kappa=2$ and $f$ is totally real.

\section{Minimal 2-spheres in $G(2,4)$ with Constant Curvature and Kaehler Angle}

In this section, we look at minimal 2-sphere $S^{2}$ in $G(2,4)$ with constant curvature and Kaehler angle. Let $f: S^{2} \rightarrow G(2,4)$ be a minimal isometric immersion. By $[4,7]$ we know that at least one of the $\partial$-transformation and the $\bar{\partial}$ transformation is degenerate. For simplicity we assume that $f$ is neither holomorphic nor anti-holomorphic. Let us say $\operatorname{rank}(\partial f)=1$ and $\operatorname{rank}(\bar{\partial} f) \geq 1$. Choosing a suitable local unitary frame $Z_{1}, Z_{2}, Z_{3}, Z_{4}$ along $f$ as before, we have

$$
A=\left(\begin{array}{cc}
a_{13} & 0 \\
0 & 0
\end{array}\right), \quad B=\left(\begin{array}{ll}
b_{13} & b_{14} \\
b_{23} & b_{24}
\end{array}\right) \text {. }
$$

By $(2.17)_{2}$ and $(4.1)$ we get

$$
\begin{gathered}
d a_{13}+a_{13}\left(\omega_{33}-\omega_{11}-\sqrt{-1} \rho\right) \equiv 0 \quad \bmod \varphi \\
\omega_{34}=a \varphi, \quad \omega_{21}=b \varphi
\end{gathered}
$$

$(4.2)_{1}$ and a result of Chern [2] show that $a_{13}$ is a function of analytic type. Since $\operatorname{rank}(\partial f)=1, a_{13}$ has only isolated zeros. $\operatorname{But} \operatorname{tr}\left(A \bar{B}^{t}\right)=a_{13} \bar{b}_{13}=0$, so $b_{13}=0$, and consequently,

$$
\omega_{13}=a_{13} \varphi, \quad \omega_{14}=b_{14} \bar{\varphi}, \quad \omega_{23}=b_{23} \bar{\varphi}, \quad \omega_{24}=b_{24} \bar{\varphi} .
$$

Now from $(2.17)_{3}$ and $(4.2)_{2}$ we have

$$
\begin{array}{r}
d b_{14}+b_{14}\left(\omega_{44}-\omega_{11}+\sqrt{-1} \rho\right) \equiv 0 \bmod \bar{\varphi} \\
d b_{23}+b_{23}\left(\omega_{33}-\omega_{22}+\sqrt{-1} \rho\right) \equiv 0 \bmod \bar{\varphi} \\
d b_{24}+b_{24}\left(\omega_{44}-\omega_{22}+\sqrt{-1} \rho\right)+\left(a b_{23}-b b_{14}\right) \varphi \equiv 0 \bmod \bar{\varphi} .
\end{array}
$$

It follows that $b_{14}$ and $b_{23}$ are functions of analytic type. By taking the exterior derivative of $(4.2)_{2}$ we obtain

$$
\begin{gathered}
d a+a\left(\omega_{44}-\omega_{33}-\sqrt{-1} \rho\right) \equiv \bar{b}_{23} b_{24} \bar{\varphi} \quad \bmod \varphi \\
d b+b\left(\omega_{11}-\omega_{22}-\sqrt{-1} \rho\right) \equiv-\bar{b}_{14} b_{24} \bar{\varphi} \quad \bmod \varphi
\end{gathered}
$$

From (4.2), (4.3) and (4.4) ${ }_{1-2}$ we can calculate out as in the proof of Lemma 3.1, 
that

$$
\begin{aligned}
& \frac{1}{4} \Delta \log \left|a_{13}\right|^{2}=\frac{1}{2} \kappa-2\left|a_{13}\right|^{2}+\left|b_{14}\right|^{2}+\left|b_{23}\right|^{2}+|a|^{2}+|b|^{2} \\
& \frac{1}{4} \Delta \log \left|b_{14}\right|^{2}=\frac{1}{2} \kappa-2\left|b_{14}\right|^{2}+\left|a_{13}\right|^{2}-\left|b_{24}\right|^{2}+|a|^{2}-|b|^{2} \\
& \frac{1}{4} \Delta \log \left|b_{23}\right|^{2}=\frac{1}{2} \kappa-2\left|b_{23}\right|^{2}+\left|a_{13}\right|^{2}-\left|b_{24}\right|^{2}-|a|^{2}+|b|^{2}
\end{aligned}
$$

from which it follows that

$$
\frac{1}{4} \Delta \log \left|b_{14} b_{23}\right|^{2}=\kappa+2\left(\left|a_{13}\right|^{2}-\left|b_{14}\right|^{2}-\left|b_{23}\right|^{2}-\left|b_{24}\right|^{2}\right)=\kappa+2 \cos \alpha
$$

Since both $\kappa$ and $\alpha$ are constant, if $b_{14} b_{23} \neq 0$, then by Lemma 4.1 of [6], there is a nonnegative integer $N$ such that

$$
-\pi N=(\kappa+2 \cos \alpha) \cdot 4 \pi / \kappa,
$$

and consequently, $\kappa=-8 \cos \alpha /(N+4)$, and of course this is possible only if $\cos \alpha<0$. If $b_{14} b_{23}=0$, we can assume that $b_{14}=0$ without loss of generality. Then in this case $(4.5)_{2}$ yields

$$
\frac{1}{4} \Delta \log |b|^{2}=\frac{1}{2} \kappa+\left|b_{23}\right|^{2}+\left|b_{24}\right|^{2}+\left|a_{13}\right|^{2}-2|b|^{2}
$$

Combining (4.6) and (4.9) we get

$$
\frac{1}{4} \Delta \log \left|a_{13} b_{23} b\right|^{2}=\frac{3}{2} \kappa,
$$

so $b b_{23}=0$ and consequently $b=0$ or $b_{23}=0$. If $b=0$, then

$$
\frac{1}{4} \Delta \log \left|a_{13} b_{23}\right|^{2}=\kappa-1
$$

which yields that $b_{23}=0$ or as in (4.8),

$$
-\pi N^{\prime}=(\kappa-1) \cdot 4 \pi / \kappa
$$

where $N^{\prime}$ is a nonnegative integer. In the latter case, $\kappa=4 /\left(N^{\prime}+4\right)$. Now we consider the case $b_{14}=b_{23}=0$. Then by $(4.4)_{3},(4.5)$ and $(4.6)_{1}$ we have

$$
0=\frac{1}{4} \Delta \log \left|a_{13}\right|^{2}=\frac{1}{2} \kappa-2\left|a_{13}\right|^{2}+|a|^{2}+|b|^{2},
$$




$$
\begin{aligned}
0=\frac{1}{4} \Delta \log \left|b_{24}\right|^{2} & =\frac{1}{2} \kappa-2\left|b_{24}\right|^{2}+|a|^{2}+|b|^{2}, \\
\frac{1}{4} \Delta \log |a|^{2} & =\frac{1}{2} \kappa+1-2|a|^{2}, \\
\frac{1}{4} \Delta \log |b|^{2} & =\frac{1}{2} \kappa+1-2|b|^{2} .
\end{aligned}
$$

It is easy to see from $(4.13)_{1-2}$ that $\left|a_{13}\right|^{2}=\left|b_{24}\right|^{2}=1 / 2$, i.e., $f$ is totally real. Now (4.13) yields

$$
\frac{1}{4} \Delta \log \left|a_{13} b_{24} a b\right|^{2}=2 \kappa
$$

so $a b=0$. Without loss of generality we assume that $a=0$, then we have

$$
\frac{1}{4} \Delta \log \left|a_{13} b_{24} b\right|^{2}=\frac{3}{2} \kappa-1
$$

Consequently, we have $b=0$ or as in the above argument, $\kappa=4 /\left(N^{\prime \prime}+6\right)$, where $N^{\prime \prime}$ is a nonnegative integer. In the case where $a=b=0$, by (4.13) we get $\kappa=2$. In summary, we have shown

THEOREM 4.1. Let $f: S^{2} \rightarrow G(2,4)$ be an isometric minimal immersion from $S^{2}$ into $G(2,4)$ with constant curvature $\kappa$ and constant Kaehler angle $\alpha$, and $\alpha \neq 0$, $\pi$. Then $\kappa=8|\cos \alpha| /(N+4)$ or $\kappa=4 /(N+4)$ or $\kappa=2$, where $N$ is a nonnegative integer. In the last case, $f$ is totally real.

COROllary 4.2. Let $f: S^{2} \rightarrow G(2,4)$ be a totally real isometric minimal immersion from $S^{2}$ into $G(2,4)$ with constant curvature $\kappa$. Then $\kappa=4 /(N+4)$ or $\kappa=2$, where $N$ is a nonnegative integer.

\section{References}

[1] Bolton J., Jensen G. R., Rigoli M. and Woodward L. M., On conformal minimal immersion of $S^{2}$ into $C P^{n}$, Math. Ann., 279 (1988), 599-620.

[2] Chern S. S., On the minimal immersions of the two-spheres in a space of constant curvature, Problems in Analysis, Princeton University Press, 1970, pp. 27-40.

[ 3 ] Chern S. S. and Wolfson J. G., Minimal surfaces by moving frames, Amer. J. Math., 105 (1983), 59-83.

[ 4 ] Chern S. S. and Wolfson J. G., Harmonic maps of the two-sphere into a complex Grassmann manifold II, Ann. of Math., 125 (1987), 301-335.

[5] Chi Q. and Zheng Y., Rigidity of pseudo-holomorphic curves of constant curvature in Grassmann manifolds, Trans. Amer. Math. Soc., 313 (1989), 393-406. 
[6] Eschenburg J. H., Guadalupe I. V. and Tribuzy R. A., The fundamental equations of minimal surfaces in $C P^{2}$, Math. Ann., 270 (1985), 571-598.

[7] Zheng Y., Quantization of curvature of harmonic two-spheres in Grassmann manifolds, Trans. Amer. Math. Soc., 316 (1989), 193-214.

\author{
Wu Bing-Ye \\ Department of Mathematics \\ Zhejiang Normal University \\ Jinhua, Zhejiang \\ 321004 P.R. China
}

